\title{
Informatics Systems and Modelling - Case Studies of Expert Interviews
}

\author{
Leopold Lehner ${ }^{1}$, Johannes Magenheim ${ }^{1}$, Wolfgang Nelles ${ }^{1}$, Thomas Rhode ${ }^{1}$, \\ Niclas Schaper ${ }^{1}$, Sigrid Schubert ${ }^{2}$, and Peer Stechert ${ }^{2}$ \\ ${ }^{1}$ University of Paderborn, Paderborn, Germany \\ \{firstname.name\}@uni-paderborn.de \\ ${ }^{2}$ University of Siegen, Siegen, Germany \\ \{firstname.name\}@uni-siegen. de
}

\begin{abstract}
This article presents the results of two case studies undertaken within the project MoKoM funded by the German Research Foundation (DFG). In this context, expert interviews were conducted in order to identify relevant competencies empirically concerning informatics comprehension and modelling. The interviews $(\mathrm{N}=30)$ were based on typical scenarios of this domain and were conducted with different expert groups (experts of informatics, experts of didactics of informatics, expert informatics teachers). The goal of the interview analyses was to exemplarily examine the competence descriptions given by the different experts with regard to the categories of a theoretically derived competence model. The competence descriptions were also compared with reference to the different expert domains. Furthermore it was tried to identify recurring response patterns in the interviews with reference to the experts' background.
\end{abstract}

Keywords: Competence Model Research, Pedagogies, Informatics System Comprehension, Informatics Modelling, Active Learning.

\section{Motivation}

This article describes the results of an exemplary comparison of expert interviews which have been conducted within the project MoKoM funded by the German Research Foundation (DFG). Generally, the project MoKoM pursues three goals: (1) developing a scientific and empirically proofed competence model concerning the two domains informatics modelling and informatics system comprehension, (2) developing and testing instruments which are appropriate for competence measurement and finally (3) designing and evaluating effective learning environments for competence development.

With regard to the shift towards output orientation of the German school system this competence model can be understood as a basis for the development of educational standards for these two mentioned domains of informatics secondary education.

Within the project MoKoM, the starting point for competence modelling was a competence framework which was developed on the basis of theoretical considerations involving expert papers and curricula. After that, an empirical approach to 
identify the relevant competencies was chosen by the means of conducting expert interviews. They represent an adequate empirical method to supplement and to refine the already existing competence framework.

30 experts on informatics, with a division in three equal groups were interviewed: (1) experts of informatics, (2) experts of didactics of informatics and (3) expert informatics teachers. Each expert interview included four hypothetic scenarios (in a total of twelve), which describe typical and challenging tasks of informatics system comprehension and modelling and served as structured starting points for the competence interviews. Based on these scenarios, the interviewees were asked to describe their personal procedures and thoughts to solve the presented informatics problems in detail as well as questions referred to specific competence requirements of the framework model to solve the problem.

The main objective of this article is to compare the three expert groups (expert of informatics, expert of didactics of informatics, expert informatics teacher) concerning their specific contributions to describe competence facets for the model. In which respect do the experts differ in their competence-relevant statements and how can these different contributions be explained with reference to different expert perspectives, backgrounds and attitudes toward the topic? In order to answer these research questions, exemplary interviews of the three expert groups with reference to two complex hypothetic scenarios were content analyzed: (1) "Merchandise Management System" which especially treats system development requirements and (2) "Testing of Unknown Software" which treats system comprehension requirements in particular. The analysis was focused on three exemplary interviews concerning each scenario.

The article presents the exemplary results of these content analyses and is subdivided as follows: Section 2 gives an overview of the theoretically derived framework of the competence model whereas first refinements could be processed by content analytical results. Section 3 and 4 focus on the two hypothetical scenarios mentioned above. They analyze the experts' described problem solving behaviour and provide information on the impact of the results on the framework. Section 5 attempts to sketch out characteristics of the different types of experts. Section 6 presents a summary and a discussion concerning the deployed research methods.

\section{Theoretically Derived Framework of the Competence Model}

The theoretically derived competence model comprises a total of four primary dimensions: (1) Basic Competencies, (2) Informatics Views, (3) Complexity, and (4) NonCognitive Skills. The first dimension includes knowledge and skill elements concerning system development, system comprehension and system application. In this context, the ability to use an informatics system (IS), the ability to comprehend the structure, processes and individual components of an IS and the capability to design and to reengineer an IS are important.

The second dimension consists of outer and inner perspectives towards an informatics system such as usability, algorithms and data structures, graphical notation techniques and so on. It is necessary for coping with complex informatics tasks and demands that learners are enabled to look at an IS from different informatics views. 


\section{K1 Basic Competencies \\ K1.1 System Application \\ K1.2 System Comprehension \\ K.1.2.1 Requirements \\ K.1.2.2 Test \\ K.1.3 System Development K1.3.1 Business Modeling K1.3.2 Requirements K1.3.3 Analysis \& Design K1.3.4 Implementation K1.3.5 Test K1.3.6 Deployment}

K2 Informatics Views
K2.1 External View
K2.1.1 Expectations of Systems'Behavior
K2.1.2 Informatics Literacy \& Professional Practice
K2.1.3 Usability
K2.1.4 Functional View
K2.2 Internal View
K2.2.1 Layered Architectures
K2.2.2 Net-Centric Computing
K2.2.3 Systems of Patterns
K2.2.4 Algorithms \& Data Structures
K2.2.5 Fundamental Ideas of Computer Science
K2.2.6 Graphical Representations
K2.2.7 Languages (Programming \& Modeling)
K2.2.8 Computional Thinking (imperative, functional,
Iogical, object-orientated)

Fig. 1. Competence Dimensions K1 (BAsic Competencies) and K2 (Informatics Views)

The third dimension deals with the demands on handling complexity of informatics systems. Requirements regarding informatics competence vary because of the complexity and intricacy of an IS. Among others, degrees of interactivity and degrees of interconnectedness are elements of this dimension.

The fourth dimension is subdivided in three categories: attitudes, socialcommunicative / cooperative skills and motivational / volitional skills. All of these skills are prerequisites for successful problem solving within the two focused domains.

\section{K3 Complexity}

K3.1 Number of Components of Informatics Systems K3.2 Level of Net-Centric Computing K3.3 Stand-alone Systems vs. Distributed Systems K3.4 Level of Human-Computer Interaction K3.5 Combinatorial Complexity K3.6 Measures of Complexity: Time \& Space K3.7 Scalabilty

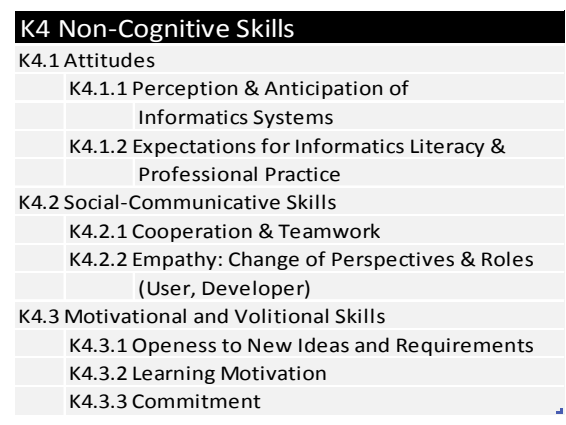

Fig. 2. Competence Dimensions K3 (COMPLEXITY) and K4 (NON-Cognitive SKILls)

These dimensions and categories were derived from curricula and expert papers covering informatics modelling and system comprehension. Thus, this step of the project MoKoM was characterized by a theoretic and deductive methodical procedure. The following step was complementary to the first one insofar as an inductive 
and empirical procedure was chosen to identify relevant competence facets by means of conducting expert interviews deploying the Critical Incident Technique.

During each interview, hypothetic informatics scenarios were presented and the interviewees were asked to give a detailed description of their problem solving approach. The interviews were audio-recorded, transcribed and analyzed by means of a qualitative content analysis method according to Mayring [1]. Using this method, the transcribed expert statements were summarized and structured with reference to the framework model categories in order to supplement and to refine our theoretically derived competence model.

Figures 1 and 2 show the four main dimensions, categories and facets of the competence model. The emphasized categories expose refinements of the competence model which were additionally integrated into the model on the bases of the first results of the content analysis.

\section{Case Study System Comprehension}

\subsection{Scenario "Testing of Unknown Software"}

In order to refine the theoretically derived competence model [2] we used three scenarios on system comprehension [3]: Scenario 4 "Usability Engineering", Scenario 5 "Computer configuration", and Scenario 7 "Car configuration". Our experience with the results of Scenario 4 has shown that this is not only a typical scenario of system comprehension, but it also contains aspects of system application. On the other hand, Scenario 5 and 7 are very similar if one is not focused on the issue of the concrete configuration software. Therefore, we recommend combining Scenarios 5 and 7 for evaluation, because they offer very similar results through qualitative content analysis.

In this paper the competence dimension K1.2 (SYSTEM COMPREHENSION) and its relations to other competence dimensions will be presented through concentration on the typical scenario "Testing of Unknown Software (developed by others)". This scenario and its questions were introduced to the interviewees as follows.

\footnotetext{
Scenario 5+7: "You are asked by a colleague to test his software, which was developed to solve configuration problems, e.g., to set up a new car or a new computer."

Question 1: "What is your strategy of testing to solve this problem? Which aspects do you have to bear in mind?"

Question 2: "Which cognitive skills are required for such a software exploration?"

Question 2.1: "Which informatics views are important for this task?"

Question 2.2: "Which complexity would you assign to this task?"

Question 3: "Which attitudes or social-communicative and cooperative skills are necessary to accomplish this?"

Question 4: "Which differences of competence levels would you expect between novices and experts?"

Question 5: "How can you as a tester be supported by this task?"

Question 6: "Could you imagine a pupil's potential procedure to solve this problem?"

Question 7: "Which obstacles would pupils have to cope with?"
}

Altogether, eight experts were interviewed with reference to such a scenario. Afterwards these interviews were analyzed with means of the above mentioned method 
of qualitative content analysis. We selected only one representative per expert group (one expert of informatics, one expert of didactics of informatics, one expert informatics teacher) to describe typical response and attitudinal patterns of these three expert groups.

In the following sub sections we discuss three phenomena. First, we discuss the empirical proof of the theoretically derived competence categories, second, empirical findings concerning additional competence categories, and third, flaws resp. problems of the interview method caused by the different meaning of scientific terms used in the interview questions.

\subsection{Basic Competencies and Non-cognitive Skills}

All three representatives of the expert groups confirmed the theoretically derived categories of K1.2 (SYSTEM COMPREHENSION) of competence dimension K1 (BASIC COMPETENCIES) with the qualitative content analysis in the following way:

Answer by an expert informatics teacher K1.2.1 (REQUIREMENTS VERSUS PERFORMANCE): "Necessary is to check requirements and to compare them with the final system."

Answer by an expert of informatics K1.2.2 (TEST): "I recommend systematic testing of software. Testing is a very difficult task, especially if the tester knows only partly the semantic relations of the functionality of the system."

Answer by an expert of didactics of informatics K1.2.3 (QUALITY OF SOFTWARE): “Inevitable is the evaluation of the quality of the software with criteria, e.g., correctness, usability, security."

For K4 (Non-Cognitive Skills) we observed response patterns, which were characteristic for our three expert groups of the interviews. The experts in informatics answered very briefly to K4. Experts in didactics of informatics deliver much more detailed statements to $\mathrm{K} 4$. But the most comprehensive version of recommendations on K4 was given by the expert informatics teachers. Some examples will illustrate this:

Answer of an expert of informatics K4.1 (Attitudes) and K4.3 (Motivational and VOLITIONAL SKILLS): "Such a person should be very ambitious to find errors. We should reward the finding of errors. This could be a kind of a competition in error detecting."

Answer of an expert informatics teacher K4.2 (Social-Communicative SkiLls): "In my opinion the ideal tester should have very good social-communicative skills. As a teacher I would expect to be asked in difficult situations. But I also appreciate a lot the teamwork between the learners of my groups. They should be able to act appropriately in different kinds of roles, e.g., as a customer, developer, and tester. A highlight of this learning process is the opportunity to present the results of the individual and team work in a plenum, to be open for critical comments and to feel responsible not to insult others through careless statements."

The presented results show that we were able to confirm and refine a theoretically derived competence model of an informatics knowledge domain by means of expert interviews (especially using the Critical Incident Technique and qualitative content analysis). This demonstrates the suitability of our research methodology. In the next sub section it is shown that the methodology is also useful to identify gaps of the theoretically derived competence categories. 


\subsection{Complexity}

We conducted the interview analysis concerning the competence dimension K3 (COMPLEXITY) in a different way (see Fig. 3, Section 2). According to a normative analysis approach using international curricula categories this procedure would lead to a overly closed focus on informatics lessons at school [4]. In contrast to this approach it would not be helpful to use all complexity categories of theoretical informatics for our competence model [5]. So we had to find an interview and analysis method between these extremes. We will illustrate this with the following statements on K3 (CompLeXITY) with our experts' groups:

Answer by an expert of informatics: "I have to deliver a perfect specification from where the tester can recognize all possible cases which he has to evaluate. If this testing scenario should be realized, you need an authentic informatics system with a realistic functionality. This task of systematic testing leads in a combinatorial complexity."

Answer by an expert of didactics of informatics: "The main challenge of this testing task is the combination of all possible cases, which the tester has to evaluate. This means he has to cope with combinatorial complexity. The testing results are depending on the resources available."

Answer by an expert informatics teacher: "Systematic testing means that you have to check each function with all possible parameters, which the software offers. The task is connected with a high complexity, because you have to handle all possible cases, which means combinatorial complexity."

The method of qualitative content analysis shows clearly that our theoretically derived competence model has to be complemented. Therefore, we added the competence category K3.5 (Combinatorial complexity) to the competence dimension K3 (Complexity). Combinatorial complexity means that all combinations of inputs and preconditions, e.g., of a certain system, have to be considered, even though exhaustive testing may not be feasible. Further studies delivered with the same method led to the addition of the following categories: K3.6 (COMPLEXITY MEASURES: TIME AND SPACE) and K3.7 (SCALABILITY). To characterize the quality of algorithms, their runtime performance (including efficiency) and memory requirements are usually evaluated in informatics. They are referred to as complexity measures, time and space. Concerning scalability we include the following characteristic of informatics: Some standard algorithms deliver good results for a small input size, but for a large input size they deliver bad results or no solution in a given time boundary. Therefore, the criterion scalability can be used to decide, which solution (system, algorithm, process) should be applied to different problem sets.

\subsection{Informatics Views}

After describing the positive effects of the applied research methodology, we will now discuss the flaws resp. problems of the empirical method. In particular, these were caused by the different meanings of some scientific terms in the different expert groups we interviewed. We discovered this methodical problem when analyzing the competence dimension K2 (INFORMATICS VIEWs). The expert of informatics was not familiar with the term "informatics views" in didactics of informatics [6] and so, he 
did not answer adequately with reference to this competence dimension. Unfortunately, the interviewers did not recognize this misunderstanding of the expert and did not clarify it in the interview.

The informatics teacher was not familiar with the term "informatics views", either. He misinterpreted the question and described the view of an informatics system from the user's perspective, i.e. the "graphical user interface" and gave a detailed description of menus and buttons for the interaction.

The expert of didactics of informatics had no problems with the term "informatics views". He answered this question in an adequately structured way: with reference to this competence dimension he differentiated between view of interaction, functional view, and view of system states. Consequently, he described the educational efforts of each recommended view with great detail.

Thus, we have to train the interviewers and the conductors of content analysis to be aware of these problems. The interviewers should be particularly sensitive to misinterpretations of a question and notice when the expert feels uncomfortable with some questions. In these cases, they have to be trained to efficiently clarify such misunderstandings or feelings of irritation. Additionally the questions of the interview guideline have to be revised concerning the use of informatics terms, which are accepted by all three groups of experts, not only by the small community of experts of didactics of informatics.

\section{Case Study Informatics Modelling}

\subsection{Scenario "Merchandise Management System"}

Analogous to the case study "System Comprehension", three scenarios were used to refine our theoretically derived competence model focusing the competence categories and facets of informatics modelling including non-cognitive aspects. These are 1 . Merchandise Management System, 2. Chat-System and 3. Web-based Game. In the following the first mentioned scenario was used for our exemplary analysis.

By means of the interviewees' answers it will be discussed how to exemplarily refine our theoretically derived competence model. Analogous to the first case study, differences between the three expert groups will be illustrated. Also, potential instructional proposals how to teach the competences - stated by the experts - will be exposed.

\footnotetext{
Scenario "Merchandise Management System": "You are asked to develop a software based merchandise management system for a small school kiosk."

Question 1: "What is your course of action to solve this task? Which software engineering workflows do you have to process?"

Question 2: "Which graphical models would you apply?"

Question 2.1: "Which informatics views are important for this task?"

Question 2.2: "Which complexity would you assign to this task?"

Question 3: "Which cognitive skills are required to develop such a software system?"

Question 4: "Could you imagine a potential pupil's procedure to solve this problem?"

Question 5: "Which attitudes, social communicative skills and motivational aspects are necessary to solve this problem?”
} 


\subsection{Basic Competencies and Non-cognitive Competencies}

In summary, the interviewees confirmed the competence category K1.3 (SYSTEM DEVELOPMENT) whereas differences in managing the software development process were observable.

The expert of informatics stated that he would prefer a software development process, which runs through several iterations. This confirms our competence category of K1.3 (System DeVELOPMENT), which was derived from the core workflows of the Rational Unified Process, recommending an iterative way of proceeding [7, p. 1]. Therefore, it seems to be appropriate to add another competence category resp. facet "Sequencing Pattern" to K1.3 (System Development). This indicates that informatics modelling covers competencies to deliberately select problem solving strategies according to the respective context (or the respective iteration) the learners are faced [8, p. 8].

In contrast, the expert informatics teacher favours a classical way of proceeding, i.e. the waterfall model. This also confirms the category of $K 1.3$ because the workflows of the waterfall model are in a way analogical to the core workflows of RUP. They could be interpreted as a static part of the RUP.

The expert of didactics of informatics just describes the requirements workflow, which confirms the respective category of the theoretically derived competence model K1.3.2 (REQUIREMENTS WORKFLOW).

Concerning the relevant phases of the software engineering process the interviewees presented different ways of proceeding: the expert of informatics and the expert of didactics of informatics stick to a well established iterative course of action whereas the expert informatics teacher prefers the classic waterfall model. This circumstance might indicate that the university teachers are more involved in best practices of modern software development.

The expert of informatics recognises software development as a communicative and co-operative process. Besides social competencies like the ability to work in teams, he emphasises communication abilities as absolutely necessary for a close cooperation between developers and customers. Both groups represent different areas of expertise, which must be brought together. Avoiding domain-specific vocabulary and the use of graphical media like object-diagrams facilitate this interchanging process: "The use of well-adapted graphical representations of object-diagrams leads to good discussions with customers and domain-experts".

Negotiation and communication skills seem to be also essential for a successful integration of 'non-informatics-persons' into the software-development-process; such social skills are also crucial for a successful performance within the developers' team.

The informatics expert points to another important social skill for the co-workers in the developer group: the ability of developing 'awareness'. To achieve this goal, the expert hints at the use of computer-based tools even at school.

For the expert of didactics of informatics, the ability to work together in teams is seen as a fundamental skill for the performance of software development projects at school, including the ability to adequately criticize others and to handle criticism.

The teachers' role is to act as an observer of the cooperation-processes. When some pupils are not able to work together in one team it may be necessary to initialize the reorganisation of the groups. 


\subsection{Complexity}

Introducing different stages of the software development process the expert of informatics provides information on complexity: Starting the software-project with textual descriptions of typical use case scenarios, he raises the question, to what (depth of) detail that should be done. He assumes that it might be very difficult, especially for beginners, to find an adequate level of granularity. He is sure that the key for a successful school project is the ability to determine an adequate requirement-definition, which later on will be implementable.

Similar to the expert of informatics the expert of didactics of informatics considers complexity as difficulty to extract elements of relevance involved in a businessprocess: "Crucial is the process of abstraction, the decision making about relevant and not relevant aspects."

According to the expert informatics teacher, it is not possible to rate the complexity of this scenario. From a modelling view it is rather useful to choose an adequate structural complexity degree for this scenario. Hence, the teachers' task is to place appropriate limitations on the range of complexity of the informatics system.

\section{Characteristics of Types of Experts}

Beyond the analysis results considered so far, we identified some recurring characteristics of the different types of experts. In the following, we give some examples.

We assumed that informatics experts would be very sceptical towards introducing some scenarios at school. However, regarding the "Merchandise Management System", we recognized a different attitude pattern concerning the appropriateness of the scenario to be used in informatics secondary education.

Answer of the expert of informatics: "A merchandise management system could be implemented at secondary level without a doubt."

Answer of the expert of didactics of informatics: "I am not able to present didactical and methodical considerations ad-hoc without any preliminary thoughts."

Answer of the expert informatics teacher: "This scenario might be appropriate for informatics secondary education although there may occur difficulties in some cases."

In this case, it was the expert of informatics that expressed not a negative but a positive attitude towards the appropriateness of the scenario for informatics secondary education - in contrast to the expert of didactics and the expert teacher, which were more critical concerning the appropriateness of the scenario. So, we have to be careful to generalize that experts of informatics are more critical concerning the schoolappropriateness of informatics learning contents. Such appraisals might also depend on the personal experiences or other background characteristics of an expert.

Furthermore our interview analyses showed that especially experts of informatics felt uncomfortable with scenarios, which covered parts of informatics, that were not in their research field. An expert of informatics in the field of algorithms and data structures stated: "I want to stress that the scenario on data base systems is beyond my experience". However, he gave valuable hints how to cope with the scenario. 
Another expert of informatics even refused to answer questions on object-oriented modelling, because he was not familiar with so called Class Responsibility Collaboration cards.

But again, this response pattern could not only be observed with reference to expert informatics. Also an expert of didactics of informatics refused to answer questions concerning the scenario "Merchandise Management System" on the fly. He stated that he has to perform a comprehensive preliminary planning before he could answer the scenarios' questions in an appropriate manner.

We also observed one expert of informatics who was very familiar with software testing who preferred to skip the scenario on software testing: "Let us skip this scenario, the requirements specification is not accurate, so I cannot choose an adequate testing strategy".

These difficulties to answer the interview questions were altogether rather exceptions. In most cases the experts were willing and able to describe in detail how they would proceed in the scenario and which competence facets are relevant in the situation.

Another characteristic of experts of didactics of informatics was that they tried to describe a vision of informatics education at schools, e.g., the necessity to teach first broad knowledge in informatics followed by concrete examples: "A necessary prerequisite to solve the problem is the understanding of fundamental mechanisms, connections and concepts".

Such experts of didactics of informatics have infrequently contact to pupils, so, sometimes, their answers how pupils would cope with a situation remained rather abstract and were not connected to a certain learning process: "It just depends what the student has learnt so far".

The more experienced the experts were in the field of school informatics, the easier we could match their meaning units with the categories of the theoretically derived competence model. More precisely, almost every expert informatics teacher saw every scenario from a teacher's point of view even though he should explain at first, how he would cope himself with the scenario. Sometimes this dilemma was pointed out before the expert answered: "Before I answer the question I would like to ask, whether I should answer from my own perspective or as a teacher in a concrete learning process?"

Other experts, though, immediately switched to the teachers point of view: "As far as I understood you, I have to realize this scenario at school. Then I would analyse the scenario and create a mind map to identify the important themes my pupils have to learn". Others supplement their own procedures with potential pupil's procedures to solve this problem even though they were not asked to do so.

In summary, we could identify some recurring characteristics of the different types of experts, which were illustrated here by examples. However, we have to be careful to generalize these findings based on the relative small amount of analysed data so far and concerning the mentioned counterexamples. It could also be, that the statements rather reflect the personal experiences or backgrounds of the very expert than the characteristics of the group he or she is representing.

\section{Summary and Conclusions}

Taken together, we can conclude that by means of these case studies we could gain some important insights into the appropriateness of the theoretically derived categories 
of the competence model of informatics modelling and comprehension. Especially, the competence dimension K1 (BASIC COMPETENCIES) with its categories K1.2 (System COMPREHENSION) and K1.3 (SYSTEM DEVELOPMENT) and their sub-categories could be confirmed by the descriptions of the experts. That is of particular importance because the dimension $\mathrm{K} 1$ is the core dimension of the entire competence model. Furthermore, the experts' answers on questions concerning social competence requirements provided valuable and confirming clues to the fourth dimension Non-Cognitive Skills. All experts generally ascribed relevance to this dimension. However, the experts' relation to the school context has apparently an impact on the degree of description of these skills: The closer the experts' relationship to school, the more differentiated the noncognitive skills are described.

Furthermore, the analyses showed that the experts' competence descriptions can also be used to derive valuable suggestions concerning the design of effective learning environments for competence development at schools. For instance, be shown for the exercising of communicative skills in system development or the limitation of the complexity of the used informatics system.

With reference to the dimension K3 (COMPLEXITY) our exemplary analysis results showed that the chosen empirical method is feasible not only to refine a theoretically derived competence model but also to adjust and complement such a model. According to this approach the sub-categories K3.5 (COMBINATORIAL COMPLEXITY), K3.6 (COMPlexity Measures): Time and Space and K 3.7 (SCAlability) were added to the model categories.

With regard to the competence dimension K2 (INFORMATICS VIEWs), also, methodical difficulties with the chosen empirical approach could be demonstrated. These were especially caused by misunderstandings of the term Informatics Views used in the interviews.

Furthermore, additional analyses with reference to response and attitude-related patterns of the specific expert groups showed that the interviewer has to be also sensitive to certain characteristics and backgrounds of the different expert groups concerning competence interviews. The presented analyses so far, display only exemplary analyses of the whole range of our interviews. So, in further steps the complete interview data have to be analyzed with the described method of content analysis. And consequently, the competence model has to be furthermore refined, adjusted and formulated.

After we have reached this point of model development we have to consider that there are further restrictions of the described empirical approach to model competencies. Firstly, this refers to the restriction of the scenario approach of the interviews. The relevant competence facets of the informatics modelling and comprehension domain might be identified only selectively by means of the scenario interviews. Secondly, the interview technique raises only verbal descriptions of how to cope with certain competence requirements. This might not cover the real requirements in every relevant aspect.

So, it is necessary to conduct additional empirical research steps to proof the content and criteria validity of the developed competence model: The evaluation of the content validity of the model can be accomplished by an expert rating. Therefore, the different informatics experts have to rate the extracted competence descriptions concerning their relevance, difficulty, representativeness and degree of differentiation. 
The evaluation of the criteria validity of the competence model can be accomplished by developing instruments to measure the different facets of the competence model and the criteria behaviour. The resulting correlations between both can be interpreted as indicators for criteria validity of the competence model.

\section{References}

1. Mayring, P.: Qualitative Inhaltsanalyse. Beltz, Weinheim (2003)

2. Kollee, C., Magenheim, J., Nelles, W., Rhode, T., Schaper, N., Schubert, S., Stechert, P.: Computer science education and key competencies. In: 9th IFIP World Conference on Computers in Education - WCCE 2009, Bento Goncalves, Brazil (2009),

http: / /www.die.informatik. uni-siegen.de/ e-publikationen/Publikationen/2009/WCCE2009_pap147.pdf

3. Stechert, P.: Fachdidaktische Diskussion von Informatiksystemen und der Kompetenzentwicklung im Informatikunterricht, Development of Competencies with Informatics Systems. In: Commentarii Informaticae Didacticae, vol. 2, Universitätsverlag Potsdam (2009)

4. Tucker, A. (ed.): A Model Curriculum for K-12 Computer Science: Final Report of the ACM K-12 Task Force Curriculum Committee, 2nd edn. ACM, New York (2006), http: / / www . csta.acm.org

5. Cross, J., Denning, P.: Computing Curriculum 2001. The Joint Curriculum Task Force IEEE-CS/ ACM Report (2001), http: / / www . computer. org/education/ cc2 001

6. Stechert, P., Schubert, S.: A strategy to structure the learning process towards understanding of informatics systems. In: Working/Joint IFIP-Conference Informatics, Mathematics and ICT (IMICT 2007), Boston, USA (2007), http://www.die.informatik.unisiegen.de/e-publikationen/Publikationen/2007/2007_Boston_ stechert_schubert.pdf

7. Rational Software Corporation IBM. Rational unified process. best practices for software development teams, white paper (1998)

8. Magenheim, J., Nelles, W., Rhode, T., Schaper, N., Schubert, S., Stechert, P.: Competencies for Informatics Systems and Modeling. Results of Qualitative Content Analysis of Expert Interviews. In: Proceedings of the 1st Global Engineering Education Conference - Educon 2010. IEEE Computer Society, Los Alamitos (2010) (in Press) 